\title{
Application of Statistical Design to Assess the Critical Process Parameters of Ethanol Injection Method for the Preparation of Liposomes
}

\author{
Sayani Bhattacharyya and Bharani S. Sogali \\ Department of Pharmaceutics, Krupanidhi College of Pharmacy, Bangalore, 560035, Karnataka, India
}

(Received: February 05, 2019; Accepted: May 22, 2019; Published (Web): June 30, 2019)

\begin{abstract}
In the present study custom screening design was employed to observe the effect of four critical process parameters on particle size and polydispersity index of the liposomal formulation made by ethanol injection method. The four process parameters selected were lipid ratio, rate of injection, phase volume ratio and rotational speed of magnetic stirring. Eight different liposomal formulations were prepared using the design. The formulations were subjected to particle size analysis. The analysis was done at a significance level $p<0.05$ and found that the process parameters had significant effect on the particle size and polydispersity index of the formulations. The design was optimized for the individual responses with an overall desirability of more than $50 \%$. Three batches of liposomes were formulated at optimized process parameters which matched the target as predicted by the design. Therefore, it can be concluded that the design was effective in production of nano sized stable monodisperse liposomes by ethanol injection method.
\end{abstract}

Key words: Liposome, ethanol injection method, critical process parameters, response, custom design

\section{INTRODUCTION}

Liposomes are sphere shaped vesicles with an enclosed aqueous layer in the concentric bilayer membrane of phospholipids. Cholesterol and natural phospholipids can be used to make these vesicles artificially. ${ }^{1}$ They are promising systems for drug delivery of both hydrophilic and hydrophobic drug. ${ }^{2}$ The amphipathic phospholipid molecules in aqueous milieu orient themselves into supramolecular selfassemblages of concentric vesicles. ${ }^{3}$ The bilayer components determine the rigidity of the membrane. Saturated phosphatidylcholine forms a rigid bilayer membrane, whereas unsaturated phosphatidylcholine makes the bilayer less stable and more permeable. ${ }^{4,5}$ Presence of cholesterol in the bilayer membrane of unsaturated phosphatidylcholine helps to make the

Correspondence to: Sayani Bhattacharyya Mobile: 919845561865

Email: sayanibh@gmail.com

Dhaka Univ. J. Pharm. Sci. 18(1): 103-111, 2019 (June) DOI: https://doi.org/10.3329/dujps.v18i1.41897 membrane more ordered, increases the phase transition temperature and gives a modulatory effect on the bilayer membrane. ${ }^{6}$ Cholesterol can be incorporated as fluidity buffer in a very high concentration of $1: 1$ to $2: 1$ molar ratio of cholesterol to phospholipids. ${ }^{7}$

There are several methods reported for preparation of liposomes. Ethanol injection method was reported by Batzri and Korn in 1973 as one of the alternative methods for preparing SUVs without sonication and regardless of drug solubility. ${ }^{8}$ It is a simple reproducible method of preparing liposomes without lipid degradation or oxidative alterations. ${ }^{9}$ In this method an ethanolic solution of lipids are rapidly injected into an aqueous solution. Lipid molecules precipitate when the ethanolic solution encounters the aqueous phase. It forms bilayer planar fragments which transforms into quasi spherical structure of liposomal system. This procedure yields a high proportion of single unilamellar vesicles (SUVs). ${ }^{10}$ The critical process parameters that can affect the 
vesicle size are varied lipids proportion in ethanol, the rate of injection of lipids, magnetic stirring speed, and the phase volume ratio of solvent and nonsolvent.

The vesicle size of liposomes has significant influence of their cellular uptake stability, biodistribution, encapsulation efficiency, drug release profile, and stability. ${ }^{11}$ Particles in the size range of $100-150 \mathrm{~nm}$ are circulated in the systemic circulation and are not eliminated by lungs, kidney and heart, whereas smaller particles 20-100 nm may distribute to bone marrow, spleen and liver sinusoids and can leave the bloodstream via the leaky capillaries of these organs to some extent. The pore size of the pulmonary capillary barrier is estimated to be approximately $35 \mathrm{~nm}$, therefore, formulations with a particle size $<5 \mu \mathrm{m}$ can be trapped by lungs alveoli. ${ }^{12}$ Therefore, the biodistribution and half life of liposomal delivery is greatly influenced by the vesicle size of the formulation.

In this study the effect of process parameters on particle size and poly dispersity index of liposomes by ethanol injection method has been emphasized through custom design of experiments using JMPV11 software.

\section{MATERIALS AND METHODS}

Materials. Soyaphosphophatidylcholine (SPC), cholesterol and $96 \%$ ethanol were purchased from Sigma Aldrich. Purified water was obtained by double distillation.

\section{Liposome preparation}

Experimental design. Preparation of liposome by ethanol injection method is a multivariate procedure and the process parameters can affect the desired property of the liposome. ${ }^{13,14}$ Four such factors like cholesterol SPC ratio (X1) rotational speed of magnetic stirrer (X2), organic and aqueous phase volume ratio (X3) and rate of injection of organic phase (X4) were evaluated for particle size analysis for the preparation of drug free liposome. A custom design with eight experiments and three replicates was constructed using software JMP version 11. Using this design, the magnitude of effect of each variable on the resulting response on particle size and poly dispersity index was estimated independently and in confounding. Each variable was tested at two levels low (-1) and high level (+1) against the measured response particle size. The cholesterol to SPC ratio was varied from 1:1 to 2:1. Magnetic stirrer rotational speed was varied from 500 to $600 \mathrm{rpm}$, phase volume ratio was varied from 0.25 to 0.5 and rate of injection was varied from 0.6 to 1.5 $\mathrm{ml} / \mathrm{min}$.

Preparation of liposome by ethanol injection method. The required amount of SPC, cholesterol $(\mathrm{CH})$ and butylated hydroxy toluene (BHT) were dissolved in ethanol in sonicator bath at $40 \pm 2^{\circ} \mathrm{C}$. The temperature of the distilled water was maintained at $80^{\circ} \mathrm{C}$. The ethanolic dispersion of lipids was injected by means of a syringe pump in a defined volume of distilled water under magnetic stirring at specific rpm. Spontaneous liposome formation took place as soon as ethanolic solution was in contact with the aqueous phase. Formation of liposome was confirmed by the opalescence of colloidal dispersion. ${ }^{14,15}$ The stirring was continued for 15 minutes at room temperature. Finally, the solvent and a part of nonsolvent were removed by rota evaporation under reduced pressure. Eight such formulations were prepared as per table 1 .

Table 1. Custom design layout.

\begin{tabular}{lcccc}
\hline Formulation code & $\begin{array}{c}\text { X1 } \\
\text { (Cholesterol :SPC } \\
\text { (lipid ratio) }\end{array}$ & $\begin{array}{c}\text { X2 } \\
\text { (Magnetic stirring } \\
\text { speed (rpm) }\end{array}$ & $\begin{array}{c}\text { X3 } \\
\text { (Phase volume } \\
\text { ratio) }\end{array}$ & $\begin{array}{c}\text { X4 } \\
\text { (Rate of injection } \\
\text { (ml/min)) }\end{array}$ \\
\hline F1 & -1 & +1 & -1 & +1 \\
F2 & +1 & +1 & -1 & -1 \\
F3 & +1 & -1 & +1 & +1 \\
F4 & -1 & +1 & +1 & -1 \\
F5 & -1 & +1 & +1 & -1 \\
F6 & +1 & -1 & +1 & +1 \\
F7 & -1 & -1 & -1 & -1 \\
F8 & +1 & +1 & -1 & -1 \\
\hline
\end{tabular}


Particle size analysis. The mean particle size and particle size distribution of liposomes were determined by Horiba SZ-100 nano particle dynamic light scattering (DLS) system. All the samples were diluted with double distilled water to get a suitable concentration for examination and every sample was measured in triplicate at a scattering angle of $90^{\circ}$ at $25.2^{\circ} \mathrm{C}$.

Surface charge determination. The surface charge of optimized liposomal formulations was measured with the laser doppler electrophoretic mobility measurements using Horiba SZ-100 at a temperature of $25^{\circ} \mathrm{C}$. All measurements were done in triplicate.

Surface morphology. The morphological characteristic of optimized liposomal formulations was determined by a scanning electron microscope (JEOL-JSM-6360, Japan). One drop of sample was placed on a slide and the sample was dried thoroughly in vacuum desiccator. The slide was attached to the specimen holder using double coated adhesive tape and gold coating under vacuum using a sputter coater (Model JFC-1100, JEOL, Japan) for 10 minutes, and then investigated at $10 \mathrm{kV}$.

Estimation of residual ethanol in prepared liposome by gas chromatography for optimized formulation. Gas chromatography was performed by isothermal method in GC 9800, Mayura Analytical Pvt. Ltd. The column used was capillary column (EC/5 Alltech part of $30 \mathrm{mts}$ length, outer diameter of $0.25 \mathrm{~mm}$ and internal diameter of $0.25 \mu \mathrm{m}$ ). The detector used was flame ionization detector. During experimentation the pressure was maintained by air, hydrogen and nitrogen at a limit air $-0.7 \mathrm{~kg} / \mathrm{cm}^{2}$, hydrogen $1 \mathrm{~kg} / \mathrm{cm}^{2}$, nitrogen $1 \mathrm{~kg} / \mathrm{cm}^{2}$ and capillary pressure $1 \mathrm{~kg} / \mathrm{cm}^{2}$. Temperature was maintained at $80^{\circ} \mathrm{C}$ at the injector and $100^{\circ} \mathrm{C}$ at the detector and oven. Nitrogen gas was used as a carrier. The injection volume was $1 \mu \mathrm{l} / \mathrm{min}$ and was injected using a hamilton syringe. Ethanol was used as a solvent and the results were analysed by using $\mathrm{ABC}$ Peak software. $^{16}$

\section{RESULTS AND DISCUSSION}

Custom designs are general, flexible, and good for routine factor screening or response optimization. The particle size was selected as response that needs to be optimized. Four critical parameters namely cholesterol and phospholipid ratio, magnetic stirring speed in rpm, rate of injection in $\mathrm{ml} / \mathrm{min}$, and phase volume ratio of solvent to non-solvent were imported as continuous factor in two levels (high and low). The main effects corresponding to the factors are used to describe the model.

Design evaluation was done by analysis of variance at significance level $p<0.05$. The design diagnostic table shows the design efficiency as shown in table 2. These efficiency measures are single numbers which quantifies the characteristics of one mathematical design. Though the maximum efficiency is 100 for any criterion but an efficiency of $100 \%$ is not possible. Therefore, efficiencies that are less than 100 may be perfectly satisfactory. ${ }^{17}$

Table 2. Design diagnostic.

\begin{tabular}{ll}
\hline Design diagnostic efficiency & \% Estimated \\
\hline D Efficiency & 89.130 \\
G Efficiency & 79.056 \\
A Efficiency & 80.357 \\
\hline
\end{tabular}

So it can be concluded that the design was suitable for screening the factors for the preparation of liposomes by ethanol injection method.

Employing the design eight batches of liposomal formulations were made. All the formulations were subjected for particle size analysis. The analysis revealed that the mean particle size was varied in the eight formulations from minimum 32 to maximum $676 \mathrm{~nm}$ and polydispersity index was varied from 0.2 to 0.51 , which indicated the effect of various process parameters in formulation of liposome by ethanol injection methodas listed in table 3 .

The data was analysed using JMP software V11 software. The actual vs. predicted plot showed the effectivity of the design and revealed by the graphs as shown in figures 1 and 2. 
Table 3. Particle size analysis of the formulations.

\begin{tabular}{lcc}
\hline Formulation code & Particle size & $\begin{array}{c}\text { Polydispersity index } \\
\text { (PDI) }\end{array}$ \\
\hline F1 & 54 & 0.236 \\
F2 & 251 & 0.5 \\
F3 & 117 & 0.4 \\
F4 & 32 & 0.2 \\
F5 & 60 & 0.25 \\
F6 & 135 & 0.43 \\
F7 & 676 & 0.51 \\
F8 & 141 & 0.45 \\
\hline
\end{tabular}

Table 4. Evaluation of optimized formulations.

\begin{tabular}{lccc}
\hline $\begin{array}{l}\text { Formulation } \\
\text { code }\end{array}$ & $\begin{array}{c}\text { Particle size } \\
(\mathrm{nm})\end{array}$ & $\begin{array}{c}\text { Zeta potential } \\
(\mathrm{mV})\end{array}$ & PDI \\
\hline OF1 & $254 \pm 9.2$ & $-31.5 \pm 2.2$ & $0.429 \pm 0.02$ \\
OF2 & $361 \pm 2.5$ & $-28 \pm 1.2$ & $0.349 \pm 0.03$ \\
OF3 & $285 \pm 3.7$ & $-29.5 \pm 4.1$ & $0.384 \pm 0.05$ \\
\hline
\end{tabular}

PDI- Poly dispersity index, All values are mean $\pm \mathrm{SD}(\mathrm{n}=3)$

One way analysis of variance (ANOVA) was used to substantiate statistical differences between groups in each series of experiment. Results with $\mathrm{p}<$ 0.05 were considered significant. The analysis of the result predicted that all the factors except lipid ratio have a significant effect on particle size and all the

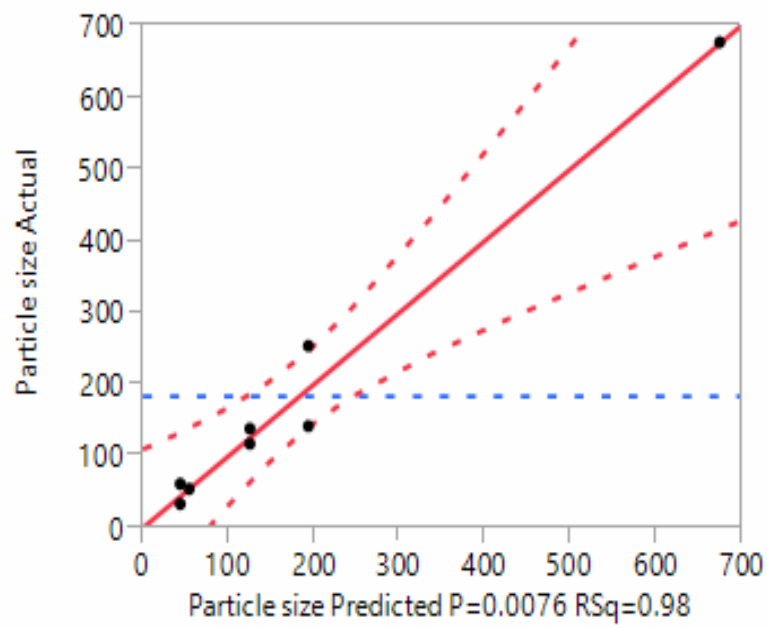

Figure 1. Actual vs predicted plot with respect to particle size. factors have significant effect on polydispersity index (PDI). The leverage plots (figures 3 and 4) showed the effect of all parameters individually with the particle size and polydispersity index.

The prediction profiler showed (figure 5) optimum (more than 50\%) desirability at magnetic stirring at $550 \mathrm{rpm}$, phase volume ratio at 0.4 , rate of injection at 0.6 (approx.) $\mathrm{ml} / \mathrm{min}$ and cholesterol SPC ratio at 1:1.5 with a predicted particle size range between $214-360 \mathrm{~nm}$ and polydispersity index range of $0.37-0.47$.

The optimized process parameters generated from the custom design were employed for formulation of liposomes. Employing the same parameters i.e., at a cholesterol to phospholipid ratio 1:1.5, rotational speed $550 \mathrm{rpm}$, rate of injection 0.6 $\mathrm{ml} / \mathrm{min}$, and a phase volume ration of 0.4 three batches of liposome (OF1, OF2 and OF3) were formulated. The formulations were subjected for the determination of particle size, zeta potential, polydispersity index, and surface morphology through SEM. The predicted and the observed particle size of the optimized formulations did not vary significantly as listed in table 4 and figures 6,7 and 8 .

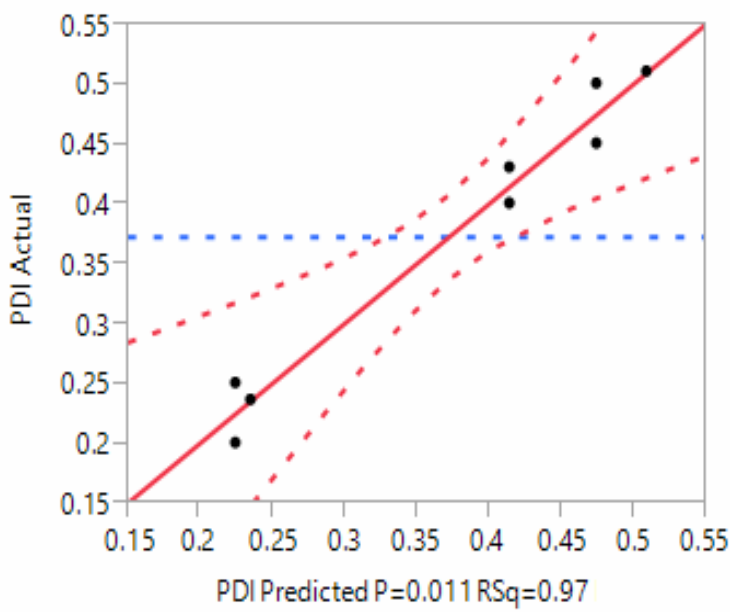

Figure 2. Actual vs predicted plot with respect to PDI. 

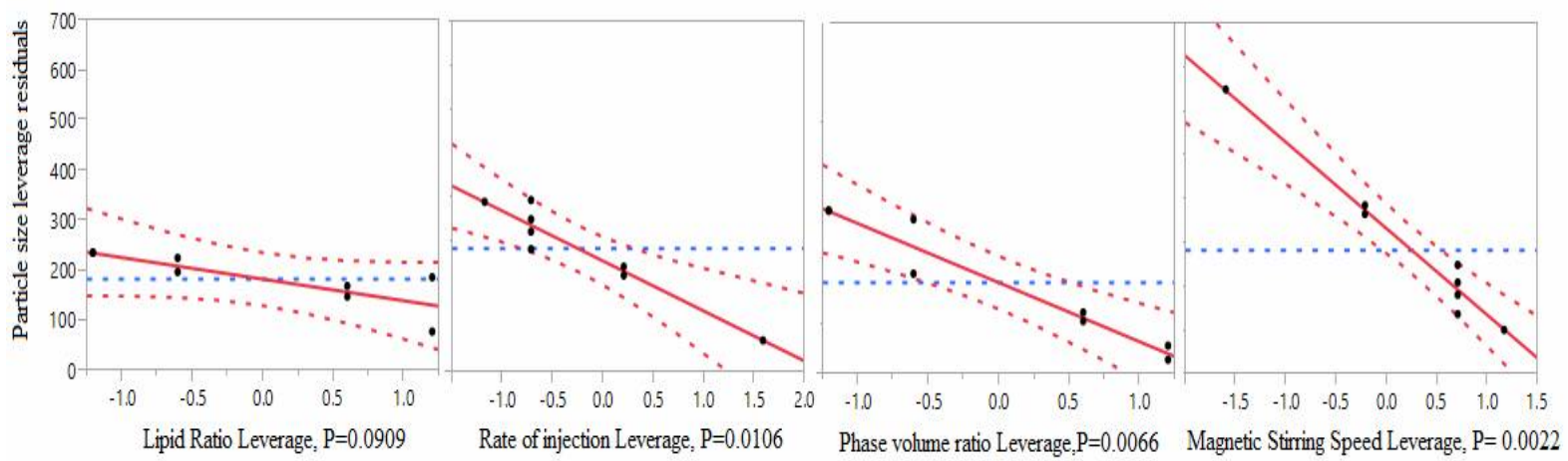

Figure 3. Leverage plots of parameters on particle size.
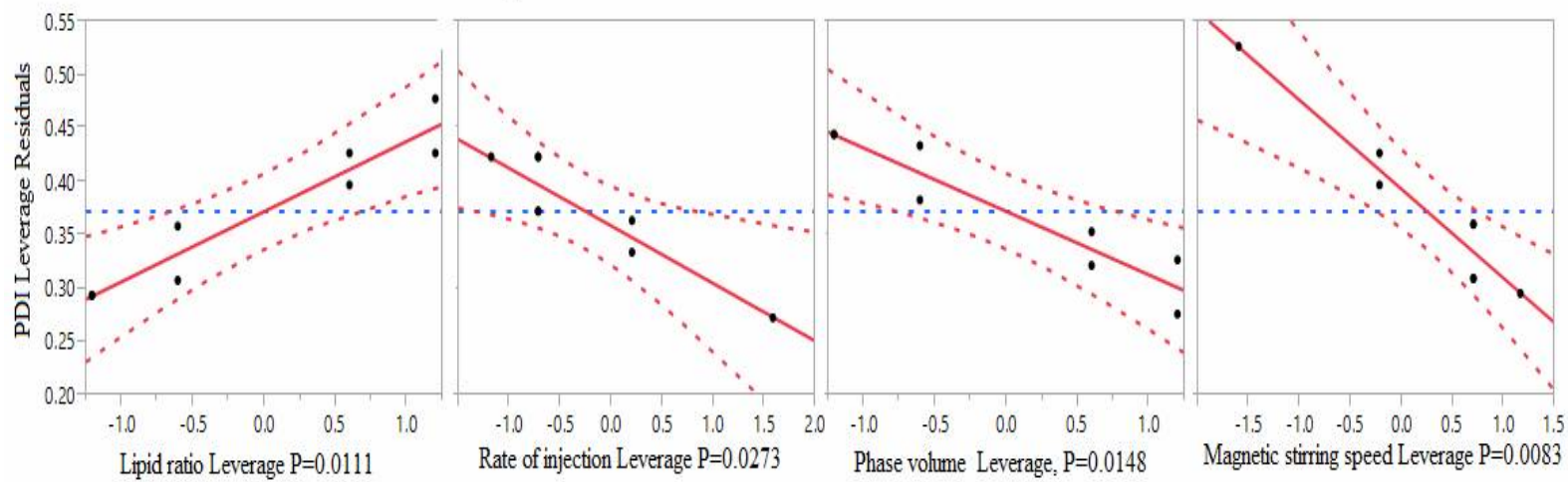

Figure 4. Leverage plots of parameters on PDI.

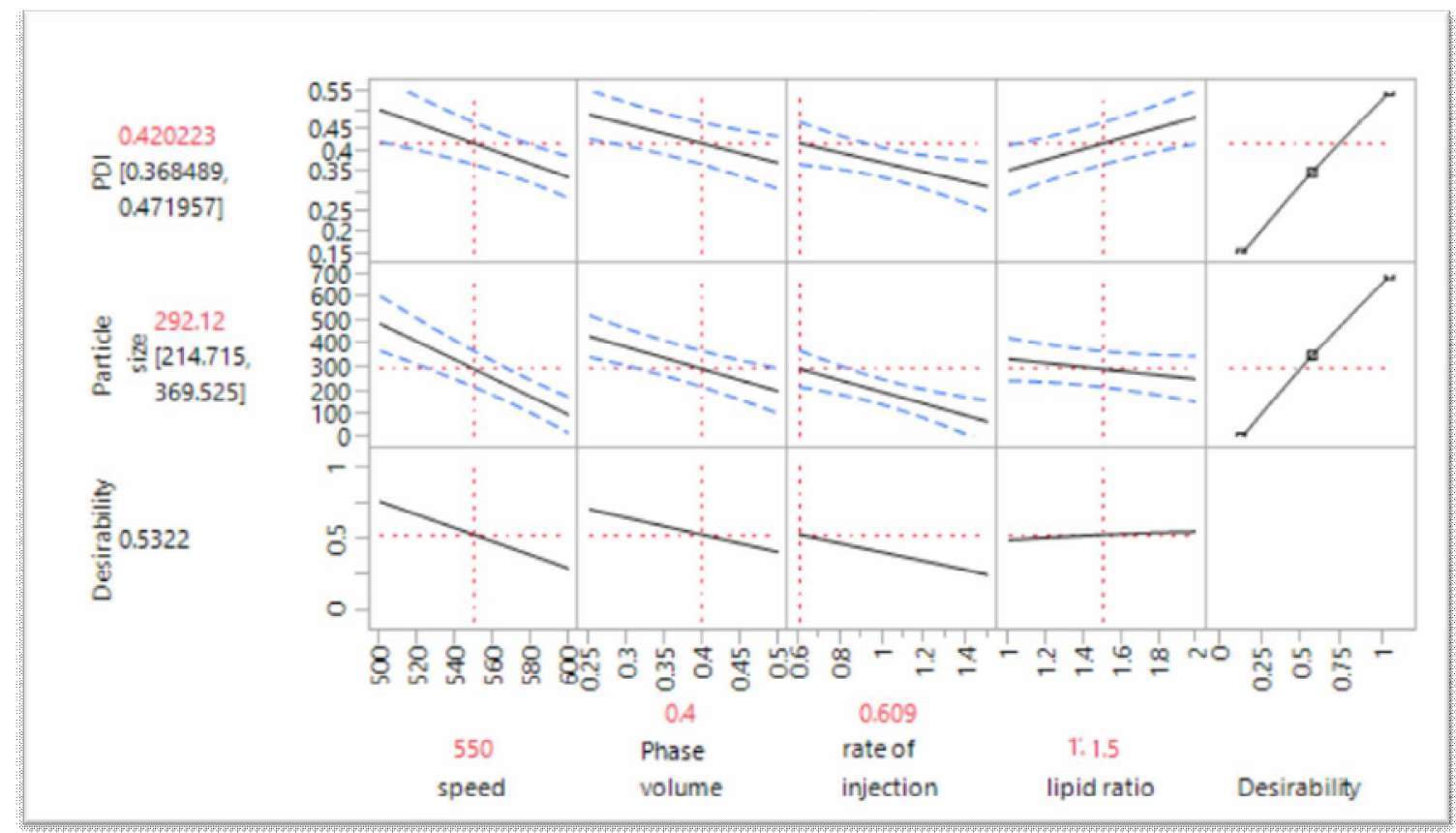

Figure 5. Prediction profiler. 

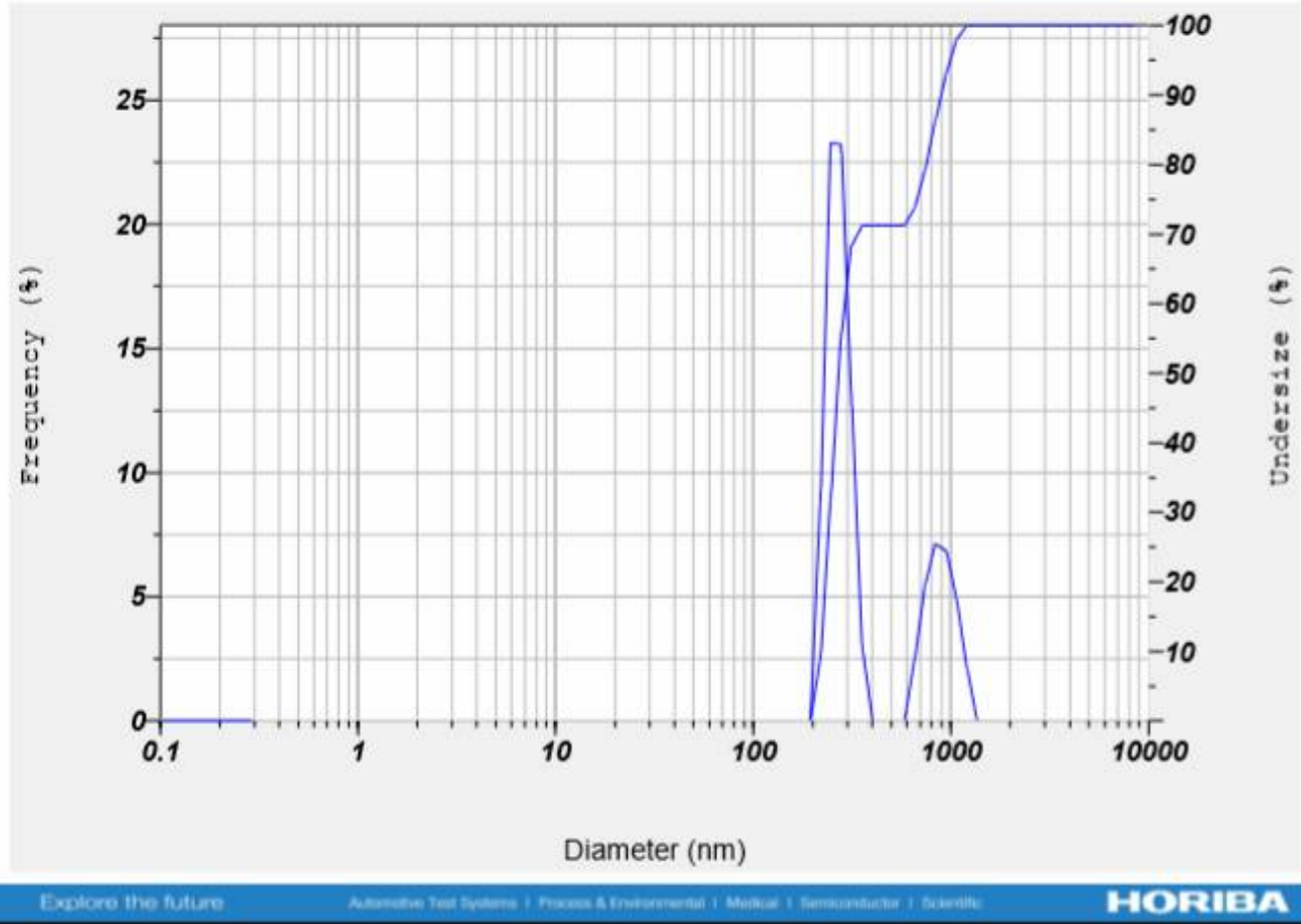

Figure 6. Particle size of optimized formulation OF1.
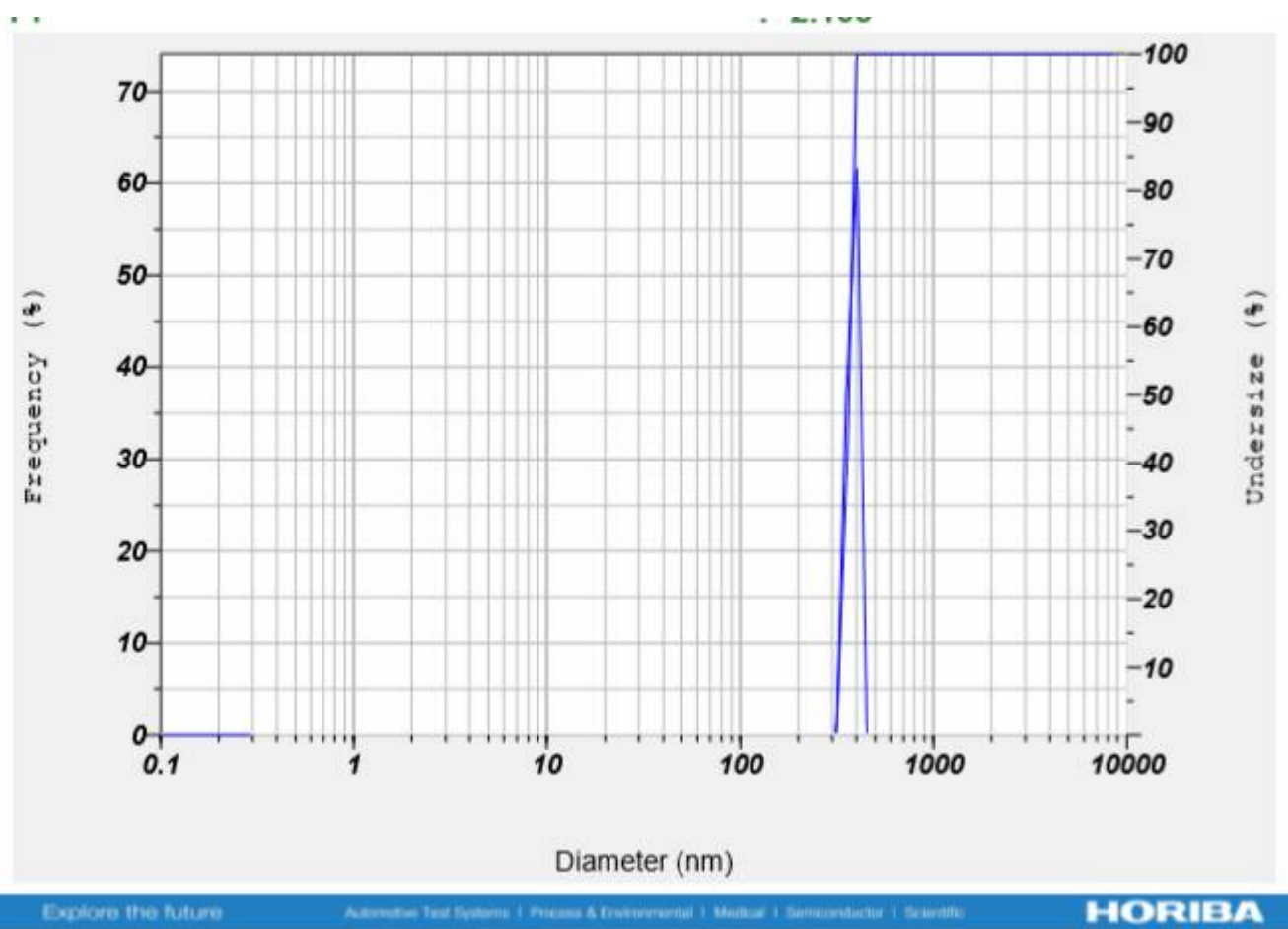

Figure 7. Particle size of optimized formulation OF2. 


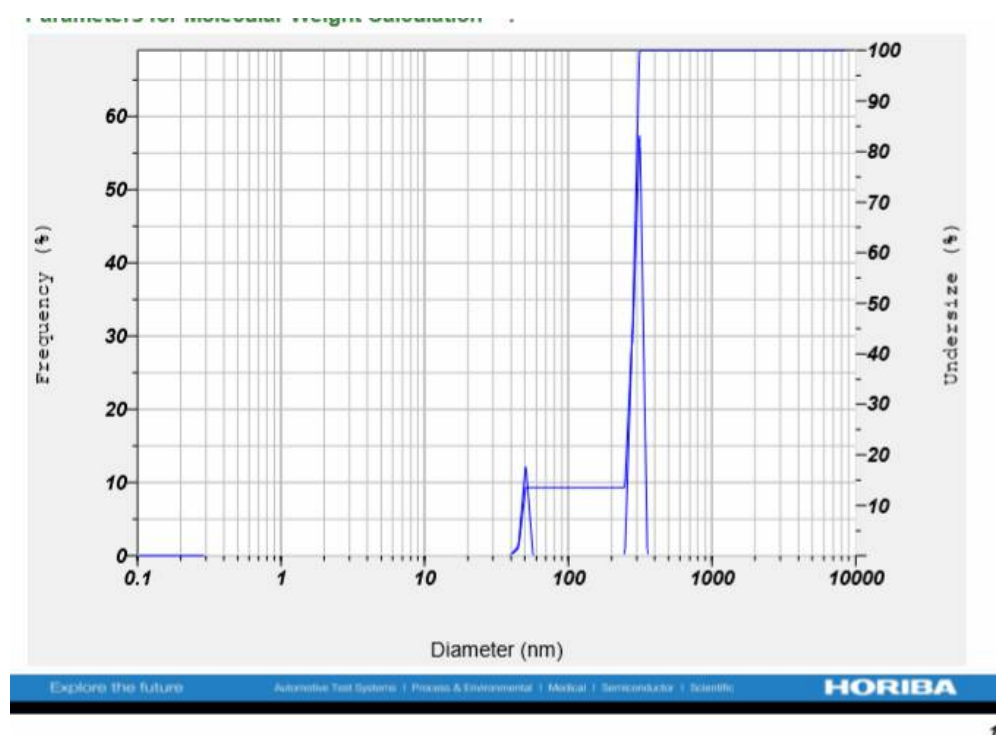

Figure 8. Particle size of optimized formulation OF3.

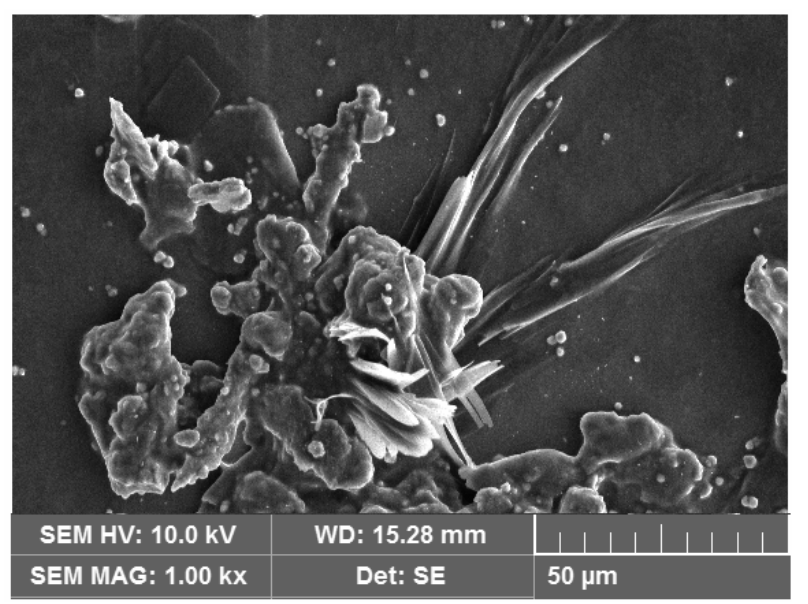

Figure 9. SEM of optimized formulation OF1.

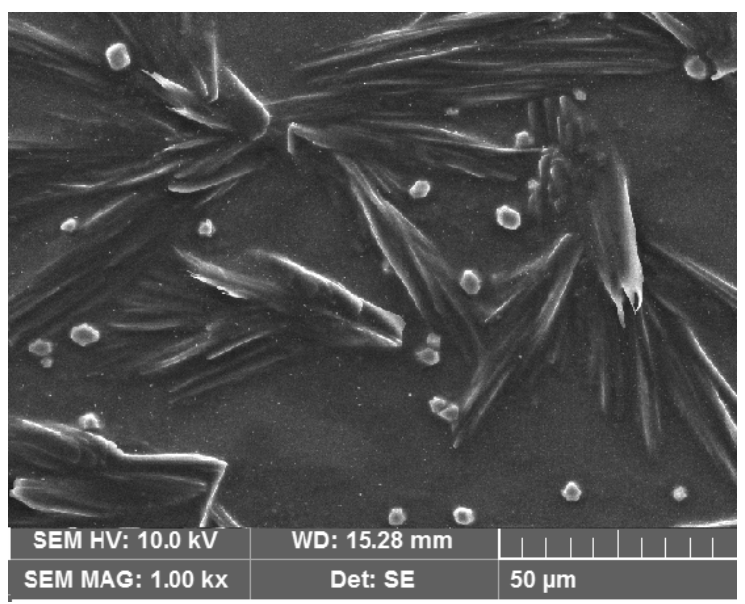

Figure 10. SEM of optimized formulation OF2.

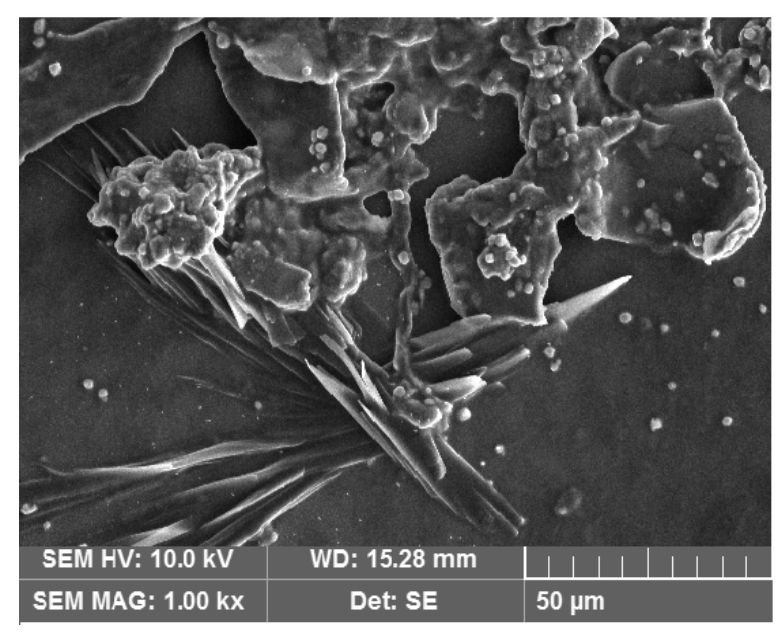

Figure 11. SEM of optimized formulation OF3. 
The polydispersity of the formulations was found to be less than 0.5 , so it can be concluded that the system was a monodisperse system. ${ }^{18}$ The surface charge was found to be within the range $-31.5 \pm 2.2$ to $-28 \pm 1.2 \mathrm{mV}$ (Table 4). The magnitude of surface charge indicated the colloidal stability of the formulations. ${ }^{19}$ The SEM photographs revealed that the liposomes were spherical and unilamellar (figures 9, 10 and 11).

The residual ethanol content in the optimized preparations was determined by gas chromatography and it was found to be $0.05 \% \mathrm{v} / \mathrm{v}$, which is within the limit as per ICH guidelines (Q3C(R6)). As per the guidelines, ethanol belongs to Class 3 low toxic potential solvents and is considered that amounts of these residual solvents of $50 \mathrm{mg}$ per day or less (corresponding to $5000 \mathrm{ppm}$ ) would be acceptable without justification. ${ }^{20}$

\section{CONCLUSION}

The custom design yielded optimized process parameters for ethanol injection method for the preparation of liposomes. The optimized process parameters found to be effective in formulation of nano sized stable liposomes. The optimized batch of liposomal formulation showed the same efficacy, reproducibility and robustness of the process. The nano sized liposomal formulations with soya phosphatidyl choline and cholesterol were found to be stable in a monodisperse system.

\section{ACKNOWLEDGEMENTS}

Authors are grateful to the management and Principal of Krupanidhi College of Pharmacy to provide us the infrastructure and support to carry out the study.

\section{Conflict of interest}

The authors confirm that this article content has no conflict of interest.

\section{REFERENCES}

1. Akbarzadeh, A., Rezaei-sadabady, R., Davaran, S., Joo, S.W., Zarghami, N., Hanifehpour, Y., Samiei, M., Kouhi, M. and Kajem, N.K. 2013. Liposome: Classification, preparation, and applications. Nanoscale Res. Lett. 8, 1-8.

2. Jaafar-Maalej, C., Diab, R., Andrieu, V., Elaissari, A. and Fessi, H. 2010. Ethanol injection method for hydrophilic and lipophilic drug-loaded liposome preparation. J. Liposome Res. 20, 228-243.

3. Lasic, D.D. 1995. Mechanisms of liposome formation. J. Liposome Res.5, 431-441.

4. Allen, T.M. 1997. Liposomes. Opportunities in drug delivery. Drugs. 54, 8-14.

5. Li, J., Wang, X., Zhang, T., Wang, C., Huang, Z., Luo, X. and Deng, Y. 2014. A review on phospholipids and their main applications in drug delivery systems. Asian. J. Pharm. Sci. 10, 81

6. Ua, J.S.,. Rana, A. C., Bhandari, A. K. and Nagle, J. 1992. Liposome: methods of preparation and applications. BBA Biomembr. III, 7.

7. Collins, J.J., Philips, M.C. 1982. The stability and structure of cholesterol-rich co dispersions of cholesterol and phosphatidylcholine. J. Lipid.. Res. 23, 291-298.

8. Shaker, S., Gardouh, A., Ghorab, M. 2017. Factors affecting liposomes particle size prepared by ethanol injection method. Res. Pharm. Sci. 12, 346-352.

9. Charcosset, C., Juban, A., Valour, J.P., Urbaniak, S. and Fessi, H. 2015. Preparation of liposomes at large scale using the ethanol injection method: effect of scale-up and injection devices. Chem. Eng. Res. Des. 94, 508-515.

10. Pons, M., Foradada, M. and Estelrich, J. 1993. Liposomes obtained by the ethanol injection method. Int. J. Pharm. 95, 51-56.

11. Bulbake, U., Doppalapudi, S., Kommineni, N. and Khan, W. 2017. Liposomal formulations in clinical use: an updated review. Pharmaceutics 9, 1-33.

12. Danaei, M., Dehghankhold, M., Ataei, S., Hasanzadeh, F., Davarani, R. Javanmard, A. Dokhani, S.K. and. Mozafari M. R. 2018. Impact of particle size and polydispersity index on the clinical applications of lipidic nanocarrier systems. Pharmaceutics 10, 1-17.

13. Porfire, A., Muntean, D.M., Rus, L., Sylvester, B. and Tomuță, I. 2017. A quality by design approach for the development of lyophilized liposomes with simvastatin. Saudi. Pharm. J. 25, 981-992.

14. Arab-tehrany, E. 2012. Optimization and characterization of liposome formulation by mixture design. Analyst 137, 773-786. 
15. Ong, S.G.M,, Ming, L.C., Lee, K.S. and Yuen, K.H. 2016. Influence of the encapsulation efficiency and size of liposome on the oral bioavailability of griseofulvin-loaded liposomes. Pharmaceutics 8. 25.

16. Puranik, S.B., Pai, R., Pai, P.N.S. and Rao, G.K. 2008. Gas chromatographic determination of residual levels of methanol and chloroform from liposomal, microspheres and nanoparticles. Int. J. Chem. Sci. 6, 693-704.

17. Marcle, P. 2009. JMP® 8 Design of Experiments Guide. $2^{\text {nd }}$ ed SAS Institute Inc., Cary, NC, USA. pp. 55-85.
18. Hadian, Z., Sahari, M.A., Moghimi, H.R. and Barzegar, M. 2014. Formulation, characterization and optimization of liposomes containing eicosapentaenoic and docosahexaenoic acids; a methodology approach. Iran. J. Pharm. Res. 13, 393 404.

19. Nanocomposix. 2012. Guideline: Zeta potential analysis of nanoparticles.. Nanocomposix Pub. 1-6.

20. ICH-Q3C(R6). 2016. International Council for Harmonisation of Technical Requirements for Pharmaceuticals for Human Use Ich Harmonised Guideline Impurities: Guideline for Residual Solvents Q3C(R6). 